\title{
Decay of Massive Scalar Hair on Brane Black Holes
}

\author{
Marek Rogatko and Agnieszka Szypłowska \\ Institute of Physics \\ Maria Curie-Sklodowska University \\ 20-031 Lublin, pl. Marii Curie-Sklodowskiej 1, Poland \\ rogat@tytan.umcs.lublin.pl \\ rogat@kft.umcs.lublin.pl
}

(Dated: November 3, 2018)

\begin{abstract}
We study analytically the intermediate and late-time behaviour of the massive scalar field in the background of static spherically symmetric brane black hole solutions. The intermediate asymptotic behaviour of scalar field reveals the dependence on the field's parameter mass as well as the multiple moment $l$, while the late-time behaviour has the power law decay rate independent on those factors.
\end{abstract}

PACS numbers: $04.50 .+\mathrm{h}$

\section{INTRODUCTION}

Recently there has been growing interests in studying models which basic idea is that our universe is only a submanifold on which the standard model is confined to inside a higher dimensional spacetime. In the above model only geometric degrees of freedom can propagate in extra dimensions. By making the volume of extra dimensions spacetime large, one was able to lower a fundamental quantum gravity scale to the electrovac scale of TeV-order. One can also construct the black hole solutions in the brane-world models. The difficulties in those attempts stem from the fact that in general brane dynamics generate Weyl curvatures which in turn backreact on the brane dynamics.

In order to simplify this attitude one can look for the analytic solutions to the projected Einstein equations on the brane Ref.[1]. Similar kind of solutions were revealed also in Refs.[2]. On the other hand, it is interesting to pose a question whether a brane on which four-dimensional black hole is situated can be found by looking for a slice that intersects a bulk black hole. It was revealed [3], [4] that brane solutions with a black hole geometry cannot be found by slicing a bulk with $G(D-2, k)$ symmetry if the brane is vacuum and not totally geodesic. In Ref. [5] a localized static but non-vacuum brane black hole solution of a slice of a $G(D-2, k)$ bulk was presented. Recently, in Ref. [6] the possibility of finding a regular Randall-Sundrum (RS) brane world on which a static spherically symmetric black hole surrounded by realistic matter is located by slicing a fixed five-dimensional bulk black hole spacetime was presented.

The decay of black hole hair is a very interesting problem on its own. Late-time behaviour of various fields in the spacetime of a collapsing body is of a great importance for black hole's physics due to the fact that regardless of details of the collapse or the structure and properties of the collapsing body the resultant black hole can be described only by few parameters such as mass, charge and angular momentum, black holes have no hair. Price in 7] for the first time studied the neutral external perturbations and found that the late-time behavior is dominated by the factor $t^{-(2 l+3)}$, for each multipole moment $l$. For the decay along null infinity and along the future event horizon it was found Ref. [8] that the power laws are of the forms $u^{-(l+2)}$ and $v^{-(l+3)}$, where $u$ and $v$ were the outgoing Eddington-Finkelstein (ED) and ingoing ED coordinates. In Ref. [9] the scalar perturbations on Reissner-Nordtröm (RN) background for the case when $|Q|<M$ was studied and it was shown the following dependence on time $t^{-(2 l+2)}$, while for $|Q|=M$ the late-time behavior at fixed $r$ is governed by $t^{-(l+2)}$. Charged hair decayed slower than a neutral one [10]- 12], while the late-time tails in gravitational collapse of a self-interacting fields in the background of Schwarzschild solution was reported by Burko [13] and in RN solution at intermediate late-time was considered in Ref. 14]. The very late-time tails of the massive scalar fields in the Schwarzschild and nearly extremal RN black holes were elaborated in Refs. [15], [16]. It was revealed that the oscillatory tail of scalar field has the decay rate of $t^{-5 / 6}$ at asymptotically late time. The power-law tails in the evolution of a charged massless scalar field around a fixed background of dilaton black hole was studied in Ref.[17], while the case of massive scalar field was treated in [18]. The problem of the late-time behaviour of massive Dirac fields were studied in the spacetime of Schwarzschild black hole [19], while in the spacetime of RN black hole was analyzed in Ref. [20].

The intense growing of interests in unification scheme such as superstring/M-theory triggered also the interests in hair decays in the spacetimes of $n$-dimensional black holes. As far as the $n$-dimensional static black holes is concerned, the no-hair theorem for them is quite well established [24]. The mechanism of decaying black hole hair in higher dimensional static black hole case concerning the evolution of massless scalar field in the $n$-dimensional Schwarzshild spacetime was determined in Ref. 25]. It was found that for odd dimensional spacetime the field decay had a power falloff like $t^{-(2 l+n-2)}$, where $n$ is the dimension of the spacetime. This tail was independent of the presence of the black hole. For even dimensions the late-time behaviour is also in the power law form but in this 
case it is due to the presence of black hole $t^{-(2 l+3 n-8)}$. The late-time tails of massive scalar fields in the spacetime of $n$-dimensional static charged black hole was elaborated in Ref.[26], where because of tremendous difficulties in solving analytically differential Eqs. of motion for the field numerical simulations for the spacetime dimension $n=5$ and $n=6$ were performed.

The main purpose of our paper will be to clarify what kind of mass-induced behaviours play the dominant role in the asymptotic late-time tails as a result of decaying the massive scalar hair in the background of brane black hole. The intermediate and late-time tails of the fields corresponding to the massive scalar ones have not been studied before in the context of brane black hole physics. On the other hand, our purpose is to see what effects of brane black hole parameters will have on the decay of the massive scalar hair on them.

The paper is organized as follows. In Sec.II we gave the analytic arguments concerning the decay of scalar massive hair in the background of the considered black holes. Sec.III will be devoted to a summary and discussion.

\section{THE DECAY OF SCALAR HAIR IN THE BACKGROUND OF BLACK HOLE BRANE SOLUTION}

\section{A. Casadio-Fabbri-Mazzacurati (CFM) brane black hole solution}

In Ref.[2] it was revealed that projecting the vacuum $n+1$-dimensional Einstein equations on a timelike manifold of codimension one leads us to the analogs of the momentum and Hamiltonian constraints in the Arnowitt-Deser-Misner (ADM) decomposition of the metric. Now their role is to select our admissible field configurations along hypersurfaces of constant coordinate. Further, it was argued that this Hamiltonian constraint is a weaker requirement than the purely $n$-dimensional vacuum Eqs. and it is equivalent to the relation for $n$-dimensional Ricci tensor $R_{i j}=E_{i j}$, where $E_{i j}$ is the projection of $n+1$-dimensional Weyl tensor on the brane 27].

Our general setting will be brane black hole solutions proposed in Ref.[2]. Two families of analytic spherically symmetric solutions with the condition that $g_{r r} \neq-\frac{1}{g_{t t}}$, parametrized by ADM mass and the post-Newtonian (PPN) parameter $\beta$ were found. The parameter $\beta$ affects the perihelion shift and the Nordtvedt effect [28].

In our considerations we shall use the general form of spherically symmetric line element

$$
d s^{2}=-A(r) d t^{2}+\frac{1}{B(r)} d r^{2}+r^{2} d \Omega^{2} .
$$

The momentum constraints are identically satisfied by the metric coefficients and the Hamiltonian constraints can be written out for them [2]. Setting $A(r)=\left(1-\frac{2 M}{r}\right)$ the resulting metric yields

$$
d s^{2}=-\left(1-\frac{2 M}{r}\right) d t^{2}+\frac{\left(1-\frac{3 M}{2 r}\right)}{\left(1-\frac{2 M}{r}\right)\left(1-\frac{\gamma M}{2 r}\right)} d r^{2}+r^{2} d \Omega^{2}
$$

where $\gamma=4 \beta-1$. One can see that for $\beta=1$ this solution reduces to the Schwarzschild black hole solution. Next to find the pattern of the decay of massive scalar hair in the underlying brane black hole spacetime we define the tortoise coordinates $y$ as

$$
d y=\frac{d r}{A(r)^{\frac{1}{2}} B(r)^{\frac{1}{2}}} .
$$

This change of variables enables one to rewrite the line element in the following form:

$$
d s^{2}=A(r)\left(-d t^{2}+d y^{2}\right)+r^{2} d \Omega^{2} .
$$

As we shall consider the scalar massive wave equation

$$
\left(\nabla_{\mu} \nabla^{\mu}-m^{2}\right) \tilde{\psi}=0
$$

in the spherically symmetric background it will be not amiss to resolve the field into spherical harmonics

$$
\tilde{\psi}=\sum_{l, \delta} \frac{1}{r} \psi_{\delta}^{l}(t, r) Y_{l}^{\delta}(\theta, \phi),
$$


where $Y_{l}^{\delta}$ is a scalar spherical harmonics on the unit two-sphere. It leads to the following equations of motion for each multipole moment

$$
\psi_{, t t}-\psi_{, y y}+V \psi=0
$$

where the effective potential $V$.

We shall analyze the time evolution of massive scalar field in the background of brane black hole by means of the spectral decomposition method. As was claimed in Refs. [14], 30] it was shown that the asymptotic tail is connected with the existence of a branch cut situated along the interval $-m \leq \omega \leq m$. An oscillatory inverse power-law behaviour of massive scalar field arises from the integral of Green function $\tilde{G}\left(y, y^{\prime} ; \omega\right)$ around branch cut. In our paper we denote it by $G_{c}\left(y, y^{\prime} ; t\right)$ and our main aim will be to find analytical form of its integral.

The time evolution of massive scalar field may be written in the following form:

$$
\psi(y, t)=\int d y^{\prime}\left[G\left(y, y^{\prime} ; t\right) \psi_{t}\left(y^{\prime}, 0\right)+G_{t}\left(y, y^{\prime} ; t\right) \psi\left(y^{\prime}, 0\right)\right],
$$

for $t>0$, where the Green's function $G\left(y, y^{\prime} ; t\right)$ is given by the relation

$$
\left[\frac{\partial^{2}}{\partial t^{2}}-\frac{\partial^{2}}{\partial y^{2}}+V\right] G\left(y, y^{\prime} ; t\right)=\delta(t) \delta\left(y-y^{\prime}\right)
$$

In what follows, our main task will be to find the brane black hole Green function. Using the Fourier transform [30] $\tilde{G}\left(y, y^{\prime} ; \omega\right)=\int_{0^{-}}^{\infty} d t G\left(y, y^{\prime} ; t\right) e^{i \omega t}$ one can reduce equation (9) to an ordinary differential equation. The Fourier's transform is well defined for $\operatorname{Im} \omega \geq 0$, while the corresponding inverse transform yields

$$
G\left(y, y^{\prime} ; t\right)=\frac{1}{2 \pi} \int_{-\infty+i \epsilon}^{\infty+i \epsilon} d \omega \tilde{G}\left(y, y^{\prime} ; \omega\right) e^{-i \omega t}
$$

for some positive number $\epsilon$. By virtue of the above the Fourier's component of the Green's function $\tilde{G}\left(y, y^{\prime} ; \omega\right)$ can be written in terms of two linearly independent solutions for homogeneous equation. Namely, one has

$$
\left(\frac{d^{2}}{d y^{2}}+\omega^{2}-V\right) \psi_{i}=0, \quad i=1,2 .
$$

As far as the boundary conditions for $\psi_{i}$ are concerned they are described by purely ingoing waves crossing the outer horizon $H_{+}$of the brane black hole $\psi_{1} \simeq e^{-i \omega y}$ as $y \rightarrow-\infty$. On the other hand, $\psi_{2}$ should be damped exponentially at $i_{+}$, namely $\psi_{2} \simeq e^{-\sqrt{m^{2}-\omega^{2}}} y$ at $y \rightarrow \infty$. A convenient form of the equation of motion for massive scalar field can be obtained by the transformation as follows:

$$
\psi_{i}=\frac{\left(1-\frac{3 M}{2 r}\right)^{\frac{1}{4}}}{\left(1-\frac{2 M}{r}\right)^{1 / 2}\left(1-\frac{\gamma M}{2 r}\right)^{1 / 4}} \xi
$$

where $i=1,2$. On expanding Eq.(11) in a power series of $M / r$ neglecting terms of order $\mathcal{O}\left((M / r)^{2}\right)$ and higher we finally achieve the relation

$$
\frac{d^{2}}{d r^{2}} \xi+\left[\omega^{2}-m^{2}+\frac{\omega^{2}\left(\frac{M}{2}(5+\gamma)\right)-m^{2}\left(\frac{M}{2}(1+\gamma)\right)}{r}-\frac{l(l+1)}{r^{2}}\right] \xi=0 .
$$

The two basic solutions which are needed to construct the Green function, with the condition that $|\omega| \geq m$ are given by $\tilde{\psi}_{1}=M_{\kappa, \tilde{\mu}}(2 \tilde{\omega} r)$ and $\tilde{\psi}_{2}=W_{\kappa, \tilde{\mu}}(2 \tilde{\omega} r)$, with the following parameters:

$$
\tilde{\mu}=\sqrt{1 / 4+l(l+1)}, \quad \kappa=\frac{\omega^{2}\left(\frac{M}{2}(5+\gamma)\right)-m^{2}\left(\frac{M}{2}(1+\gamma)\right)}{2 \tilde{\omega}} \quad \tilde{\omega}^{2}=m^{2}-\omega^{2} .
$$

Consequently it leads to the following spectral Green function:

$$
\begin{aligned}
G_{c}\left(y, y^{\prime} ; t\right) & =\frac{1}{2 \pi} \int_{-m}^{m} d w\left[\frac{\tilde{\psi}_{1}\left(y, \tilde{\omega} e^{\pi i}\right) \tilde{\psi}_{2}\left(y^{\prime}, \tilde{\omega} e^{\pi i}\right)}{W\left(\tilde{\omega} e^{\pi i}\right)}-\frac{\tilde{\psi}_{1}(y, \tilde{\omega}) \tilde{\psi}_{2}\left(y^{\prime}, \tilde{\omega}\right)}{W(\tilde{\omega})}\right] e^{-i w t} \\
& =\frac{1}{2 \pi} \int_{-m}^{m} d w f(\tilde{\omega}) e^{-i w t}
\end{aligned}
$$


First we shall take into account the intermediate asymptotic behaviour of the massive scalar field. The range of parameters are $M \ll r \ll t \ll M /(m M)^{2}$. The intermediate asymptotic contribution to the Green function integral gives the frequency equal to $\tilde{\omega}=\mathcal{O}(\sqrt{m / t})$, which in turns implies that $\kappa \ll 1$. Having in mind that $\kappa$ results from the $1 / r$ term in the massive scalar field equation of motion, it depicts the effect of backscattering off the spacetime curvature and in the case under consideration the backscattering is negligible. Finally we are left with the result

$$
f(\tilde{\omega})=\frac{2^{2 \tilde{\mu}-1} \Gamma(-2 \tilde{\mu}) \Gamma\left(\frac{1}{2}+\tilde{\mu}\right)}{\tilde{\mu} \Gamma(2 \tilde{\mu}) \Gamma\left(\frac{1}{2}-\tilde{\mu}\right)}\left[1+e^{(2 \tilde{\mu}+1) \pi i}\right]\left(r^{\prime} r\right)^{\frac{1}{2}+\tilde{\mu}} \tilde{\omega}^{2 \tilde{\mu}}
$$

where we have used the fact that $\tilde{\omega} r \ll 1$ and the form of $f(\tilde{\omega})$ can be approximated by means of the fact that $M(a, b, z)=1$ as $z$ tends to zero. The resulting Green function reduces to the following:

$$
G_{c}\left(r, r^{\prime} ; t\right)=\frac{2^{3 \tilde{\mu}-\frac{3}{2}}}{\tilde{\mu} \sqrt{\pi}} \frac{\Gamma(-2 \tilde{\mu}) \Gamma\left(\frac{1}{2}+\tilde{\mu}\right) \Gamma(\tilde{\mu}+1)}{\tilde{\mu} \Gamma(2 \tilde{\mu}) \Gamma\left(\frac{1}{2}-\tilde{\mu}\right)}\left(1+e^{(2 \tilde{\mu}+1) \pi i}\right)\left(r r^{\prime}\right)^{\frac{1}{2}+\tilde{\mu}}\left(\frac{m}{t}\right)^{\frac{1}{2}+\tilde{\mu}} J_{\frac{1}{2}+\tilde{\mu}}(m t) .
$$

Taking into account the limit when $t \gg 1 / m$ one draws the conclusion that the spectral Green function implies

$$
G_{c}\left(r, r^{\prime} ; t\right)=\frac{2^{3 \tilde{\mu}-1}}{\tilde{\mu} \sqrt{\pi}} \frac{\Gamma(-2 \tilde{\mu}) \Gamma\left(\frac{1}{2}+\tilde{\mu}\right) \Gamma(\tilde{\mu}+1)}{\tilde{\mu} \Gamma(2 \tilde{\mu}) \Gamma\left(\frac{1}{2}-\tilde{\mu}\right)}\left(1+e^{(2 \tilde{\mu}+1) \pi i}\right)\left(r r^{\prime}\right)^{\frac{1}{2}+\tilde{\mu}} m^{\tilde{\mu}} t^{-1-\tilde{\mu}} \cos \left(m t-\frac{\pi}{2}(\tilde{\mu}+1)\right) .
$$

Eq.(18) depicts the oscillatory inverse power-law behaviour. In our case the intermediate times of the power-law tail depends only on $\tilde{\mu}$ which in turn is a function of the multiple moment $l$.

However, the different pattern of decay is expected when $\kappa \gg 1$, for the late-time behaviour, when the backscattering off the curvature is important. Consequently, $f(\tilde{\omega})$ when $\kappa \gg 1$ may be rewritten in the following form:

$$
\begin{aligned}
f(\tilde{\omega}) & =\frac{\Gamma(1+2 \tilde{\mu}) \Gamma(1-2 \tilde{\mu})}{2 \tilde{\mu}}\left(r r^{\prime}\right)^{\frac{1}{2}}\left[J_{2 \tilde{\mu}}(\sqrt{8 \kappa \tilde{\omega} r}) J_{-2 \tilde{\mu}}\left(\sqrt{8 \kappa \tilde{\omega} r^{\prime}}\right)-I_{2 \tilde{\mu}}(\sqrt{8 \kappa \tilde{\omega} r}) I_{-2 \tilde{\mu}}\left(\sqrt{8 \kappa \tilde{\omega} r^{\prime}}\right)\right] \\
& +\frac{(\Gamma(1+2 \tilde{\mu}))^{2} \Gamma(-2 \tilde{\mu}) \Gamma\left(\frac{1}{2}+\tilde{\mu}-\kappa\right)}{2 \tilde{\mu} \Gamma(2 \tilde{\mu}) \Gamma\left(\frac{1}{2}-\tilde{\mu}-\kappa\right)}\left(r r^{\prime}\right)^{\frac{1}{2}} \kappa^{-2 \tilde{\mu}}\left[J_{2 \tilde{\mu}}(\sqrt{8 \kappa \tilde{\omega} r}) J_{2 \tilde{\mu}}\left(\sqrt{8 \kappa \tilde{\omega} r^{\prime}}\right)\right. \\
& \left.+e^{(2 \tilde{\mu}+1)} I_{2 \tilde{\mu}}(\sqrt{8 \kappa \tilde{\omega} r}) I_{2 \tilde{\mu}}\left(\sqrt{8 \kappa \tilde{\omega} r^{\prime}}\right)\right]
\end{aligned}
$$

where we have used the limit $M_{\kappa, \tilde{\mu}}(2 \tilde{\omega} r) \approx \Gamma(1+2 \tilde{\mu})(2 \tilde{\omega} r)^{\frac{1}{2}} \kappa^{-\tilde{\mu}} J_{\tilde{\mu}}(\sqrt{8 \kappa \tilde{\omega} r})$ 31. The first part of the above Eq. (19]) the late time tail is proportional to $t^{-1}$ and it occurs that we shall concentrate on the second term of the right-hand side of Eq.(19). For the case when $\kappa \gg 1$ it can be brought to the form written as

$$
G_{c(2)}\left(r, r^{\prime} ; t\right)=\frac{M}{2 \pi} \int_{-m}^{m} d w e^{i(2 \pi \kappa-w t)} e^{i \phi}
$$

where we have defined

$$
e^{i \phi}=\frac{1+(-1)^{2 \tilde{\mu}} e^{-2 \pi i \kappa}}{1+(-1)^{2 \tilde{\mu}} e^{2 \pi i \kappa}}
$$

while $M$ provides the relation as follows:

$$
M=\frac{(\Gamma(1+2 \tilde{\mu}))^{2} \Gamma(-2 \tilde{\mu})}{2 \tilde{\mu} \Gamma(2 \tilde{\mu})}\left(r r^{\prime}\right)^{\frac{1}{2}}\left[J_{2 \tilde{\mu}}(\sqrt{8 \kappa \tilde{\omega} r}) J_{2 \tilde{\mu}}\left(\sqrt{8 \kappa \tilde{\omega} r^{\prime}}\right)+I_{2 \tilde{\mu}}(\sqrt{8 \kappa \tilde{\omega} r}) I_{2 \tilde{\mu}}\left(\sqrt{8 \kappa \tilde{\omega} r^{\prime}}\right)\right] .
$$

At very late time both terms $e^{i w t}$ and $e^{2 \pi \kappa}$ are rapidly oscillating. It means that the scalar waves are mixed states consisting of the states with multipole phases backscattered by spacetime curvature. Most of them cancel with each others which have the inverse phase. In such a case, one can find the value of $G_{c}(2)$ by means of the saddle point method. It could be found that the value $2 \pi \kappa-w t$ is stationary at the value of $w$ equal to the following:

$$
a_{0}=\left[\frac{\pi\left(\omega^{2}\left(\frac{M}{2}(5+\gamma)\right)-m^{2}\left(\frac{M}{2}(1+\gamma)\right)\right)}{2 \sqrt{2} m}\right]^{\frac{1}{3}}
$$


By virtue of saddle point method, on evaluating the adequate expressions, we derive the resultant spectral Green function

$$
G_{c}\left(r, r^{\prime} ; t\right)=2 \sqrt{6} m^{2 / 3}(\pi)^{\frac{5}{6}}\left[2 M m^{2}\right]^{\frac{1}{3}}(m t)^{-\frac{5}{6}} \sin (m t) \tilde{\psi}(r, m) \tilde{\psi}\left(r^{\prime}, m\right),
$$

One can observe that the dominant role in the late-time behaviour plays the term proportional to $-5 / 6$.

The other case can be obtained if one considers $B(r)=\left(1-\frac{2 \gamma M}{r}\right)$ for the other model of brane black hole. It implies the following line element:

$$
d s^{2}=-\frac{1}{\gamma^{2}}\left(\gamma-1+\sqrt{1-\frac{2 \gamma M}{r}}\right)^{2} d t^{2}+\frac{d r^{2}}{\left(1-\frac{2 \gamma M}{r}\right)}+r^{2} d \Omega^{2} .
$$

For this case $\gamma=2 \beta-1$. For $\beta=1$ one has also the Schwarzschild limit.

It is convenient to introduce the coordinate changes in the manner

$$
\psi_{i}=\frac{\gamma^{\frac{1}{2}} \xi}{\left(\gamma-1+\sqrt{1-\frac{2 \gamma M}{r}}\right)^{\frac{1}{2}}\left(1-\frac{2 \gamma M}{r}\right)^{\frac{1}{4}}}
$$

where $i=1,2$, Let us expand Eq.(11) as a power series of $M / r$ neglecting terms of order $\mathcal{O}\left((M / r)^{2}\right)$ and higher. It yields

$$
\frac{d^{2}}{d r^{2}} \xi+\left[\omega^{2} \gamma^{2} \rho^{2}-m^{2}+\frac{\omega^{2}\left(4 \gamma M\left(1+\rho^{2}\right)\right)-m^{2} 2 M \gamma}{r}-\frac{l(l+1)}{r^{2}}\right] \xi=0
$$

where $\rho^{2}=(\gamma-1)^{2}+3$.

Eq. (27) can be brought to the form of the Whittaker's one with the two basic solutions are needed to construct the Green function, with the condition that $|\omega| \geq m$, i.e., $\tilde{\psi}_{1}=M_{\kappa, \tilde{\mu}}(2 \tilde{\omega} r)$ and $\tilde{\psi}_{2}=W_{\kappa, \tilde{\mu}}(2 \tilde{\omega} r)$. The parameters of Whittaker's functions implies

$$
\tilde{\mu}=\sqrt{1 / 4+l(l+1)}, \quad \kappa=\frac{\omega^{2}\left(4 \gamma M\left(1+\rho^{2}\right)\right)-m^{2} 2 M \gamma}{2 \tilde{\omega}} \quad \tilde{\omega}^{2}=m^{2}-\omega^{2} \gamma^{2} \rho^{2} .
$$

It is easy to see from preceding case that the stationarity of $2 \pi \kappa-\omega t$ can be obtained for the parameter equal to

$$
a_{0}=\left[\frac{\pi\left(\omega^{2}\left(\frac{M}{2}(5+\gamma)\right)-m^{2}\left(\frac{M}{2}(1+\gamma)\right)\right)}{2 \sqrt{2} m}\right]^{\frac{1}{3}} .
$$

Finally, the asymptotic late-time Green function reads

$$
G_{c}\left(r, r^{\prime} ; t\right)=\frac{2 \sqrt{6} m^{2 / 3}(\pi)^{\frac{5}{6}}}{\gamma^{2}\left[(\gamma-1)^{2}+3\right]}\left[m^{2}\left(4 \gamma M(\gamma-1)^{2}+14 \gamma M\right)\right]^{\frac{1}{3}}(m t)^{-\frac{5}{6}} \sin (m t) \tilde{\psi}(r, m) \tilde{\psi}\left(r^{\prime}, m\right)
$$

One can observe that as in previous cases the dominant role in the asymptotic late-time decay of SI scalar hair in the spacetime of brane black hole plays the oscillatory tail with the decay rate proportional to $t^{-5 / 6}$.

\section{B. Dadhich-Maartens-Papadopoulous-Rezania (DMPR) brane black hole solution}

One can also mention the other case of the static spherically symmetric black hole localized on a three-brane in five-dimensional gravity in Randall-Sundrum model 29]. Taking into account the effective field equations on the brane one gets the following brane black hole metric [1]:

$$
d s^{2}=-\left(1-\frac{2 M}{M_{p}^{2} r}+\frac{q^{2}}{\tilde{M}_{p}^{2} r^{2}}\right) d t^{2}+\frac{d r^{2}}{\left(1-\frac{2 M}{M_{p}^{2} r}+\frac{q^{2}}{\tilde{M}_{p}^{2} r^{2}}\right)}+r^{2} d \Omega^{2},
$$


where $q$ is a dimensionless tidal parameter arising from the projection onto the brane of the gravitational field in the bulk, $M_{p}$ is a fundamental five-dimensional Planck mass while $M_{p}$ is the effective Planck mass in the brane world. Typically, one has that $\tilde{M}_{p} \ll M_{p}$. In what follows we shall concentrate on the negative tidal charge which is claimed [1] to be the more natural case. Thus, the roots of $g_{00}=0$ are respectively $r_{+}$and $r_{-}$, namely

$$
r_{ \pm}=\frac{M}{\tilde{M}_{p}^{2}}\left(1 \pm \sqrt{1-\frac{q M_{p}^{4}}{M^{2} \tilde{M}_{p}^{2}}}\right)=M\left(1 \pm \sqrt{1+\frac{Q}{M^{2}}}\right)
$$

where $q=Q \tilde{M}_{p}^{2}$.

Let us assume further that the observer and the initial data are situated far away from the considered black hole. On evaluating what follows, it is convenient to make the change of variables. Namely, it implies

$$
\psi_{i}=\frac{\xi}{\left(1-\frac{r_{+}}{r}\right)^{1 / 2}\left(1-\frac{r_{-}}{r}\right)^{1 / 2}},
$$

where $i=1,2$. In terms of the new variables we expand Eq.(11) as a power series of $r_{ \pm} / r$ neglecting terms of order $\mathcal{O}\left(\left(r_{ \pm} / r\right)^{2}\right)$ and higher. Thus it provides the following:

$$
\frac{d^{2}}{d r^{2}} \xi+\left[\omega^{2}-m^{2}+\frac{\left(2 \omega^{2}-m^{2}\right)\left(r_{+}+r_{-}\right)}{r}-\frac{l(l+1)}{r^{2}}\right] \xi=0 .
$$

Equation (34) may be solved by means of Whittaker's functions. Just two basic solutions are needed to construct the Green function, with the condition that $|\omega| \geq m$, i.e., $\tilde{\psi}_{1}=M_{\kappa, \tilde{\mu}}(2 \tilde{\omega} r)$ and $\tilde{\psi}_{2}=W_{\kappa, \tilde{\mu}}(2 \tilde{\omega} r)$. The parameters of them are given in the manner

$$
\tilde{\mu}=\sqrt{1 / 4+l(l+1)}, \quad \kappa=\left(r_{+}+r_{-}\right)\left(\frac{m^{2}}{2 \tilde{\omega}}-\tilde{\omega}\right), \quad \tilde{\omega}^{2}=m^{2}-\omega^{2} .
$$

The preceding section arguments can be repeated providing the form of $G_{c}\left(r, r^{\prime} ; t\right)$ for the intermediate late-time decay of massive scalar hair with new Whittaker's function parameters given by relation (35). One can also see that the intermediate late-time decay of hair does not depend on brane black hole parameters but it depends only on the scalar field mass and the multipole moment $l$. As far as the late-time behaviour is concerned the spectral Green function for the late-time behaviour of massive scalar field in the brane black hole spacetime can be obtained using reasoning presented in Ref.[32], putting coupling constant in dilaton gravity $\alpha=1$ and taking the exact values of $r_{+}, r_{-}$. Namely it can be written as

$$
G_{c}\left(r, r^{\prime} ; t\right)=\sqrt{2} m^{4 / 3}(\pi)^{\frac{5}{6}}(M)^{\frac{1}{3}}(m t)^{-\frac{5}{6}} \sin (m t) \tilde{\psi}(r, m) \tilde{\psi}\left(r^{\prime}, m\right),
$$

\section{CONCLUSIONS}

In our paper we have elaborated the problem of the asymptotic tail behaviour of self-interacting scalar fields in the background of various types of brane black holes. We are interested what kind of mass-induced behaviours play the dominant role in the asymptotic intermediate and late-time tails result from the decay of massive hair on the considered brane black holes. In our research we took two kinds of brane black hole solutions presented in Refs. [1] and [2]. In the case of intermediate asymptotic behaviour we obtained the oscillatory power law dependence which in turn depend on the multiple number of the wave mode as well as the filed parameter $m$. However, as in ordinary black hole analysis, this is not the final pattern of decay rate. The decay rate which is the same for all kinds of black holes occurs at very late times. It stems from the resonance backscattering off the spacetime curvature. This decay rate is independent on the angular momentum parameter $l$ as well as the mass of hair on the brane black hole.

Our main result is that the asymptotic late-time behaviuor is of the form $t^{-5 / 6}$, exactly the same as for static spherically symmetric black holes in Einstein or modified Einstein gravity (it happened that in dilaton gravity with arbitrary coupling constant $\alpha$ the late-time behaviour is of the same form [32]). Having in mind the late-time decay rate of massive scalar fields in the background of static spherically symmetric black holes related to Einstein-Maxwell theory or the low-energy string theory, this kind of behaviour should be more or less expected. It will be not amiss to investigate the decay rate of black hair connected with other spins. The investigations in this direction is in progress and will be published elsewhere. 


\section{Acknowledgments}

This work was partially financed by the budget funds in 2007 as the research project.

[1] N.Dadhich, R.Maartens, P.Papadopoulos, and V.Rezania, Phys. Lett. B 487, 1 (2000).

[2] R.Casadio, A.Fabbri, and L.Mazzacurati, Phys. Rev. D 65, 084040 (2002),

C.Germani and R.Maartens, ibid. 64, 124010 (2001).

[3] H.Kodama, Prog. Theor. Phys. 108, 253 (2002).

[4] T.Tanaka, Prog. Theor. Phys. Suppl. 148, 307 (2003).

[5] S.Seahra, Phys. Rev. D 71, 084020 (2005).

[6] C.Galfard, C.Germani, and A.Ishibashi, Phys. Rev. D 73, 064014 (2006).

[7] R.H.Price, Phys. Rev. D 5, 2419 (1972).

[8] C.Gundlach, R.H.Price and J.Pullin, Phys. Rev. D 49, 883 (1994).

[9] J.Bicak, Gen. Rel. Grav. 3, 331 (1972).

[10] S.Hod and T.Piran, Phys. Rev. D 58, 024017 (1998).

[11] S.Hod and T.Piran, Phys. Rev. D 58, 024018 (1998).

[12] S.Hod and T.Piran, Phys. Rev. D 58, 024019 (1998).

[13] L.M.Burko, Abstracts of plenary talks and contributed papers, 15th International Conference on General Relativity and Gravitation, Pune, 1997, p.143, unpublished.

[14] S.Hod and T.Piran, Phys. Rev. D 58, 044018 (1998).

[15] H.Koyama and A.Tomimatsu, Phys. Rev. D 63, 064032 (2001).

[16] H.Koyama and A.Tomimatsu, Phys. Rev. D 64, 044014 (2001).

[17] R.Moderski and M.Rogatko, Phys. Rev. D 63, 084014 (2001).

[18] R.Moderski and M.Rogatko, Phys. Rev. D 64, 044024 (2001).

[19] J.L.Jing, Phys. Rev. D 70, 065004 (2004).

[20] J.L.Jing, Phys. Rev. D 72, 027501 (2005).

[21] L.M.Burko and G.Khanna, Phys. Rev. D 70, 044018 (2004).

[22] X.He and J.L.Jing, Nucl. Phys. B 755, 313 (2006).

[23] R.A.Konoplya, C.Molina, and A.Zhidenko, Phys. Rev. D 75, 084004 (2007).

[24] G.W.Gibbons, D.Ida and T.Shiromizu, Prog. Theor. Phys. Suppl. 148, 284 (2003), G.W.Gibbons, D.Ida and T.Shiromizu, Phys. Rev. Lett. 89, 041101 (2002), G.W.Gibbons, D.Ida and T.Shiromizu, Phys. Rev. D 66, 044010 (2002),

M.Rogatko, Class. Quantum Grav. 19, L151 (2002),

M.Rogatko, Phys. Rev. D 67, 084025 (2003),

M.Rogatko, Phys. Rev. D 70, 044023 (2004),

M.Rogatko, Phys. Rev. D 71, 024031 (2005),

M.Rogatko, Phys. Rev. D 73, 124027 (2006).

[25] V.Cardoso, S.Yoshida and O.J.C.Dias, Phys. Rev. D 68, 061503 (2003).

[26] R.Moderski and M.Rogatko, Phys. Rev. D 72, 044027 (2005).

[27] T.Shiromizu, K.Maeda, and M.Sasaki, Phys. Rev. D 62, 024012 (2000).

[28] C.M.Will, Theory and Experiment in Gravitational Physics, (Cambridge University Press, Cambridge, England, 1993).

[29] L.Randall and R.Sundrum, Phys. Rev. Lett. 83, 3370 (1999).

[30] E.W.Leaver, Phys. Rev. D 34, 384 (1986).

[31] Handbook of Mathematical Functions, edited by M.Abramowitz and I.A.Stegun, (Dover, New York, 1970).

[32] M.Rogatko, Phys. Rev. D 75, 104006 (2007). 